\title{
ON THE GENERALIZED POWER TRANSFORMATION OF LEFT TRUNCATED NORMAL DISTRIBUTION
}

\section{Okoli Christian Odilichukwu ${ }^{1}$, Nwosu Dozie Felix ${ }^{2 *}$, Osuji George Amaeze ${ }^{3}$, and Nsiegbe Nelson Anayo ${ }^{1}$}

\author{
${ }^{1}$ Department of Mathematics, Chukwuemeka Odumegwu Ojukwu University, Uli, Nigeria \\ ${ }^{2}$ Department of Mathematics and Statistics, Federal Polythenic Nekede, Owerri, Nigeria \\ ${ }^{3}$ Department of Statistics, Nnamdi Azikiwe University, Awka, Nigeria \\ *Corresponding Email: fedocon2003@gmail.com
}

Cite this article:

Okoli C.O., Nwosu D.F., Osuji G.A., Nsiegbe N.A. (2021), On the Generalized Power Transformation of Left Truncated Normal Distribution. African Journal of Mathematics and Statistics Studies 4(2), 101-116. DOI: 10.52589/AJMSS4CEW6PBO.

\section{Manuscript History}

Received: 26 June 2021

Accepted: 21 July 2021

Published: 17 Aug 2021

Copyright $\odot 2020$ The Author(s). This is an Open Access article distributed under the terms of Creative Commons AttributionNonCommercial-NoDerivatives 4.0 International (CC BY-NC-ND 4.0 ), which permits anyone to share, use, reproduce and redistribute in any medium, provided the original author and source are credited.
ABSTRACT: In this study, we considered various transformation problems for a left-truncated normal distribution recently announced by several researchers and then possibly seek to establish a unified approach to such transformation problems for certain type of random variable and their associated probability density functions in the generalized setting. The results presented in this research, actually unify, improve and as well trivialized the results recently announced by these researchers in the literature, particularly for a random variable that follows a left-truncated normal distribution. Furthermore, we employed the concept of approximation theory to establish the existence of the optimal value $y_{\max }$ in the interval denoted by $\left(\sigma_{a}, \sigma_{b}\right)\left(\left(\sigma_{p}, \sigma_{q}\right)\right)$ corresponding to the so-called interval of normality estimated by these authors in the literature using the Monte carol simulation method.

KEYWORDS: Truncated Distribution, Normal Distribution, Transformation, Moments. 


\section{INTRODUCTION AND PRELIMINARY}

Let $\omega$ be an element of an appropriate non-empty sample space $\Omega$ and $X: \Omega \rightarrow \mathrm{R}(R=$ $(-\infty, \infty))$ a real-valued function (random variable) defined on $\Omega$. To eachelement of the event

$$
\Gamma_{X}=\{\omega \in \Omega: X(\omega)=x\} \in 2^{\Omega}
$$

is associated with a probability measure $P: 2^{\Omega} \rightarrow[0,1]$ in the measure space $\left(\Omega, 2^{\Omega}, P\right)$ and then denotes the probability density function (pdf) $f$ associated with the real-valued function (random variable) $X$ by $f(x)$, where $f: \mathrm{X}(\Omega) \rightarrow[0,1]$.

Let $\alpha$ be an arbitrary but fixed point of a scalar field $\mathcal{F}$ (i.e $\alpha \in \mathcal{F}$ ), then we consider a continuous bijective function or transformation $h_{\alpha}: X(\Omega) \rightarrow$ Rdefine by

$$
h_{\alpha}(x)=x^{\alpha} \forall \alpha \in D
$$

If $f_{h_{\alpha}}$ is the function induced by $h_{\alpha}$ on $f$, thenwe denoted the probability density function (pdf) $g$ associated with the real-valued function (random variable) $h_{\alpha}$ by $f_{h_{\alpha}}(x) ; f_{h_{\alpha}}$ is the probability density function induced by $h_{\alpha}$ on $f$ such that

$$
g: \mathrm{X}(\Omega)=f_{h_{\alpha}}: \mathrm{X}(\Omega)=f: h_{\alpha}(\mathrm{X}(\Omega)) \rightarrow[0,1]
$$

\section{Remark 1.1}

(1). If $\alpha=0$, then $h_{\alpha}$ (i.e. $h_{0}$ )reduces to a constant function. Hence at this point the domain of $g$ reduces to a singleton set which is not of interest (in terms of data transformations).

(2). If $\alpha=1$, then $h_{\alpha}$ (i.e. $h_{1}$ )reduces to a identity function so that $g(x)=f(x) \forall x \in \mathrm{X}(\Omega)$.

Hence in this research, we require that $\alpha \neq 0$, as such we consider the following propositions:

\section{Proposition 1.2}

If $\alpha \leq-1$, then $g$ is an inverse $\alpha$-power transform of $f$.

\section{Proof.}

This easily follows from the fact that $h_{\alpha}(x)=\frac{1}{x^{\alpha}} \forall \alpha \geq 1$.

\section{Proposition 1.3}

If $\alpha \geq 1$, then $g$ is an $\alpha$-power transform of $f$. 
Proof.

This easily follows from the fact that $h_{\alpha}(x)=x^{\alpha} \forall \alpha \geq 1$.

\section{Proposition 1.4}

If $0<\alpha<1$, thenthere exist a positive constant $c$ such that $g$ is $\mathrm{a}(c+1)$ th root power transformation of $f$.

\section{Proof.}

If $0<\alpha<1$, then it follows that $\frac{1}{\alpha}>1 ; \Rightarrow \frac{1}{\alpha}=1+c$, for some $c>0$;

$\Rightarrow \alpha=\frac{1}{1+c}$, for some $c>0$, so that $h_{\alpha}(x)=x^{\frac{1}{1+c}} \forall c>0$ which is as stated.

\section{Proposition 1.5}

If $-1<\alpha<0$, thenthere exist a positive constantc such that $g$ is an inverse $(c+1)$ th root power transform of $f$.

\section{Proof.}

If $-1<\alpha<0$, then it follows that $0<-\alpha<1 ; \Rightarrow 0<\beta<1$, where $\beta=-\alpha$. Thus by proposition $1.4 \beta=\frac{1}{1+c}$, for some $c>0$;

$\Rightarrow \alpha=\frac{-1}{1+c}$, for some $c>0$ which is as stated.

\section{Remark 1.6}

Now, observe in particular;

(i) In proposition 1.2 , if $\alpha=-1,-2$, then $g$ is an inverse, inverse square, transform of $f$ respectively.

(ii) In proposition 1.3 , if $\alpha=1,2$, then $g$ is the identity, square, transform of $f$ respectively.

(iii) In proposition 1.4 , if $c=1, \Longrightarrow \alpha=\frac{1}{2}$, then $g$ is a square root transform of $f$.

(iv) In proposition 1.5, if $c=1, \Longrightarrow \alpha=-\frac{1}{2}$, then $g$ is an inverse square root transform of $f$. 


\section{The Left Truncated Normal Distribution}

Definition 2.1. Let $X$ be a random variable that follow a normal distribution with $\mu(\mu \neq 0)$ and variance $\sigma^{2}\left(\sigma^{2}>0\right)\left(i . e . X \sim\left(\mu, \sigma^{2}\right)\right)$ then the probability distribution function $(p d f)[15]$ is given by

$$
f(x ; \mu, \sigma)=\frac{1}{\sigma \sqrt{2 \pi}} e^{\frac{-1}{2}\left(\frac{x-\mu}{\sigma}\right)^{2}}, x \in R
$$

Lifetime data pertain to the lifetimes of units, either industrial or biological, an industrial or a biological unit cannot be in operation forever. Such a unit cannot continue to operate in the same condition forever. Any random variable is said to be truncated if it can be observed over part of its range. Truncation occurs in various situations. For example, right truncation occurs in the study of life testing and reliability of items such as an electronic component, light bulbs, etc. Left truncation arises because, in many situations, failure of a unit is observed only if it fails after a certain period (for more on this, see $[1,12]$ and the references therein). Unfortunately, often time in practice, the random variable $X$ which follow a $\mathrm{N}\left(\mu, \sigma^{2}\right)$ distribution do not take values that are less than or equal to zero $(X \leq 0)$. As such, it naturally calls for one to truncate the $p d f$ in (4) to take care of the restriction of the random variable in the region $X>0$ without alteration to the properties of the $p d f$. Hence we seek for such truncated normal distribution of $f$ and then denote it by $f_{T}$. It suffices to find a constant $M$ such that $\int_{0}^{\infty} M f(x) d x=1$, where $M$ is the so-called normalizing constant and then define $f_{T}(x)=$ $M f(x)$.

Now, we solve for such $M$ by evaluating the integral $\int_{0}^{\infty} f(x) d x$. Observe that If we take $z=$ $\frac{x-\mu}{\sigma}$, then

$$
\int_{0}^{\infty} f(x) d x=\int_{0}^{\infty} \frac{1}{\sigma \sqrt{2 \pi}} e^{\frac{-1}{2}\left(\frac{x-\mu}{\sigma}\right)^{2}} d x=\int_{\frac{-\mu}{\sigma}}^{\infty} \frac{1}{\sqrt{2 \pi}} e^{\frac{-1}{2} z^{2}} d z=\Phi\left(\frac{-\mu}{\sigma}\right)
$$

It then follows that $M=\frac{1}{\Phi\left(\frac{-\mu}{\sigma}\right)}$. Hence, the left truncated normal distribution of $f$ is given by

$$
f_{T}(x ; \mu, \sigma)=\frac{1}{\sigma \sqrt{2 \pi} \Phi\left(\frac{-\mu}{\sigma}\right)} e^{\frac{-1}{2}\left(\frac{x-\mu}{\sigma}\right)^{2}}, x \in R_{+}
$$

Observe that $0 \leq f_{T}(x ; \mu, \sigma) \leq 1 \forall x \in R_{+}\left(R_{+}=(0, \infty)\right)$ and by the method of derivation of $f_{T}(x ; \mu, \sigma)$, we have that $\int_{0}^{\infty} f_{T}(x ; \mu, \sigma) d x=1$. Thus $f_{T}(x ; \mu, \sigma)$ is a proper $p d f$.

\section{Distribution Associated with Truncated Normal Distribution under Arbitrary $\alpha$-Power Transformation}

Let $\alpha$ be an arbitrary but fixed point of a scalar field $\mathcal{F}($ i.e $\alpha \in \mathcal{F})$ and $h_{\alpha}(x)=x^{\alpha} \forall \alpha \in \mathcal{F}$ as in equation (2). There is no loss of generality if we put $y=h_{\alpha}(x)$ and $\alpha=n ; \Rightarrow y=x^{n}$. Hence by standard result in classical calculus [5], the transformed function $g$ induced by $h_{\alpha}$ on 
$f$ is given by

$$
g(y ; \mu, \sigma, n)=f_{T}(x ; \mu, \sigma)|| \frac{d x}{d y}||
$$

Where ||$\frac{d x}{d y} \|$ is the absolute value of the Jacobian (determinant) of the transformation [5]. If $y=x^{n}$, then

$$
d y=n x^{n-1} \mathrm{dx} ; \Rightarrow|| \frac{d x}{d y}||=\frac{1}{|n| x^{n-1}}
$$

By substituting appropriately into equation (6) and simplifying, we have

$$
g(y ; \mu, \sigma, n)=\left\{\begin{array}{c}
\frac{y^{\frac{1}{n}-1}}{|n| \sigma \sqrt{2 \pi} \Phi\left(\frac{-\mu}{\sigma}\right)} e^{\frac{-1}{2}\left(\frac{y^{\frac{1}{n}}-\mu}{\sigma}\right)^{2}}, y \in R_{+}, n \in \mathcal{F} . \\
0 \text { otherwise }
\end{array}\right.
$$

It now remain to show that $g(y ; \mu, \sigma, n)$ given in equation (7) is a well-defined $p d f$. It suffices to show that $\int_{0}^{\infty} g(y ; \mu, \sigma, n) d y=1$. To see this we proceed as follows:

$$
\begin{gathered}
\int_{0}^{\infty} g(y ; \mu, \sigma, n) d y=\int_{0}^{\infty} \frac{y^{\frac{1}{n}-1}}{|n| \sigma \sqrt{2 \pi} \Phi\left(\frac{-\mu}{\sigma}\right)} e^{\frac{-1}{2}\left(\frac{y^{\frac{1}{n}}-\mu}{\sigma}\right)^{2}} d y=\int_{0}^{\infty} K y^{\frac{1}{n}-1} e^{\frac{-1}{2}\left(\frac{y^{\frac{1}{n}}-\mu}{\sigma}\right)^{2}} d y ; K \\
=\frac{1}{|n| \sigma \sqrt{2 \pi} \Phi\left(\frac{-\mu}{\sigma}\right)}
\end{gathered}
$$

Let $u=y^{\frac{1}{n}} ; \Rightarrow d y=n y^{1-\frac{1}{n}}$ du, substituting into the integral above gives

$$
\int_{0}^{\infty} K y^{\frac{1}{n}-1} e^{\frac{-1}{2}\left(\frac{u-\mu}{\sigma}\right)^{2}} n y^{1-\frac{1}{n}} \mathrm{du}=\int_{0}^{\infty} n K e^{\frac{-1}{2}\left(\frac{u-\mu}{\sigma}\right)^{2}} \mathrm{du}
$$

Let $z=\frac{u-\mu}{\sigma} ; \Rightarrow \sigma d z=d u$, substituting into the integral above gives

$$
\begin{gathered}
\int_{\frac{-\mu}{\sigma}}^{\infty} n \sigma K e^{\frac{-1}{2} z^{2}} \mathrm{dz}=\int_{\frac{-\mu}{\sigma}}^{\infty} \frac{1}{\sqrt{2 \pi} \Phi\left(\frac{-\mu}{\sigma}\right)} e^{\frac{-1}{2} z^{2}} \mathrm{dz}=\left(\frac{1}{\Phi\left(\frac{-\mu}{\sigma}\right)}\right)\left(\frac{1}{\sqrt{2 \pi}} \int_{\frac{-\mu}{\sigma}}^{\infty} e^{\frac{-1}{2} z^{2}} \mathrm{dz}\right)=\frac{\Phi\left(\frac{-\mu}{\sigma}\right)}{\Phi\left(\frac{-\mu}{\sigma}\right)} \\
=1
\end{gathered}
$$

This is as required. 


\section{The $j t h$ Moment about the Mean and the Origin}

In this section, for all fixed $n \in R$, we solved for the $j t h$ moment of the random variable $Y$ about the mean $\mu$, which is also called the $j$ th central moment is defined as $\mu_{j}(\mu, \sigma, n)=$ $E\left[(Y-\mu)^{j} ; \mu, \sigma, n\right]\left(\mu_{j}(n)\right.$ for short $)$. This implies that

$$
\begin{aligned}
& \mu_{j}(n)=\int_{0}^{\infty}(y-\mu)^{j} \frac{y^{\frac{1}{n}-1}}{|n| \sigma \sqrt{2 \pi} \Phi\left(\frac{-\mu}{\sigma}\right)} e^{\frac{-1}{2}\left(\frac{y^{\frac{1}{n}}-\mu}{\sigma}\right)^{2}} d y \\
= & \int_{0}^{\infty} \sum_{p=0}^{j}(-1)^{j-p}\left(\begin{array}{c}
j \\
j-p
\end{array}\right) \mu^{j-p} y^{p} \frac{y^{\frac{1}{n}-1}}{|n| \sigma \sqrt{2 \pi} \Phi\left(\frac{-\mu}{\sigma}\right)} e^{\frac{-1}{2}\left(\frac{y^{\frac{1}{n}}-\mu}{\sigma}\right)^{2}} d y \\
= & \sum_{p=0}^{j}(-1)^{j-p}\left(\begin{array}{c}
j \\
j-p
\end{array}\right) \mu^{j-p} \int_{0}^{\infty} \frac{y^{p+\frac{1}{n}-1}}{|n| \sigma \sqrt{2 \pi} \Phi\left(\frac{-\mu}{\sigma}\right)} e^{\frac{-1}{2}\left(\frac{y^{\frac{1}{n}}-\mu}{\sigma}\right)^{2}} d y \\
= & \sum_{p=0}^{j}(-1)^{j-p}\left(\begin{array}{c}
j \\
j-p
\end{array}\right) \mu^{j-p} E\left[Y^{P} ; \mu, \sigma, n\right]
\end{aligned}
$$

and we proceed to compute the pth moment about the origin $E\left[Y^{P} ; \mu, \sigma, n\right]$ which is given by

$$
E\left[Y^{P} ; \mu, \sigma, n\right]=\int_{0}^{\infty} y^{p} \frac{y^{\frac{1}{n}-1}}{|n| \sigma \sqrt{2 \pi} \Phi\left(\frac{-\mu}{\sigma}\right)} e^{\frac{-1}{2}\left(\frac{y^{\frac{1}{n}}-\mu}{\sigma}\right)^{2}} d y=K \int_{0}^{\infty} y^{p+\frac{1}{n}-1} e^{\frac{-1}{2}\left(\frac{y^{\frac{1}{n}}-\mu}{\sigma}\right)^{2}} d y
$$

Let $u=y^{\frac{1}{n}} ; \Rightarrow d y=n y^{1-\frac{1}{n}}$ du, substituting into the integral above and simplifying, we have

$$
\begin{gathered}
K \int_{0}^{\infty} y^{p+\frac{1}{n}-1} e^{\frac{-1}{2}\left(\frac{y^{\frac{1}{n}}-\mu}{\sigma}\right)^{2}} n y^{1-\frac{1}{n}} \mathrm{du}=n K \int_{0}^{\infty} u^{\mathrm{n} p} e^{\frac{-1}{2 \sigma}\left(u^{2}-2 u+1\right)} \mathrm{du}=n K e^{\frac{-1}{2 \sigma^{2}}} \int_{0}^{\infty} u^{\mathrm{n} p} e^{\frac{-u^{2}}{2 \sigma^{2}}} e^{\frac{u}{\sigma^{2}}} \mathrm{du} \\
=n K e^{\frac{-1}{2 \sigma}} \int_{0}^{\infty} u^{\mathrm{n} p} e^{\frac{-u^{2}}{2 \sigma^{2}}} \sum_{r \geq 0} \frac{\left(\frac{u}{\sigma^{2}}\right)^{r}}{r !} \mathrm{du}
\end{gathered}
$$

Observe that the series $\sum_{r \geq 0} \frac{\left(\frac{u}{\sigma^{2}}\right)^{r}}{r !}$ converges uniformly (by ratio test) [3,13], hence by Taylors series expansion, for some positive constant $k$ (sufficiently large enough) [3,13], there exists a number $\delta\left(r_{k}\right)$ between 0 and $\frac{u}{\sigma^{2}}$ such that $\delta\left(r_{k}\right) \rightarrow 0$ as $r \rightarrow \infty$, it then follows that as $r \rightarrow \infty$

$$
n K e^{\frac{-1}{2 \sigma^{2}}} \int_{0}^{\infty} u^{\mathrm{n} p} e^{\frac{-u^{2}}{2 \sigma^{2}}} \sum_{r=0}^{k}\left(\frac{1}{r !}\left(\frac{u}{\sigma^{2}}\right)^{r}+\frac{1}{r !}\left(\frac{u}{\sigma^{2}}\right)\left(\delta\left(r_{k}\right)\right)^{r}\right) \mathrm{du}
$$


can be approximated by

$$
n K e^{\frac{-1}{2 \sigma^{2}}} \int_{0}^{\infty} u^{\mathrm{n} p} e^{\frac{-u^{2}}{2 \sigma^{2}}} \sum_{r=0}^{k} \frac{1}{r !}\left(\frac{u}{\sigma^{2}}\right)^{r} \mathrm{du}=n K e^{\frac{-1}{2 \sigma^{2}}} \sum_{r=0}^{k} \frac{1}{\sigma^{2 r} r !} \int_{0}^{\infty} u^{\mathrm{r}+\mathrm{n} p} e^{\frac{-u^{2}}{2 \sigma^{2}}} d u
$$

Let $w=\frac{u^{2}}{2 \sigma^{2}} ; \Rightarrow \sigma^{2} d w=u d u$, then substituting appropriately into the integral above and simplifying, we have

$$
\begin{aligned}
& n K e^{\frac{-1}{2 \sigma^{2}}} \sum_{r=0}^{k} \frac{1}{\sigma^{2 r} r !} \sigma^{2} \int_{0}^{\infty} \sigma^{\mathrm{r}+\mathrm{n} p-1}(2 w)^{\frac{\mathrm{r}+\mathrm{n} p-1}{2}} e^{-w} d u=
\end{aligned}
$$

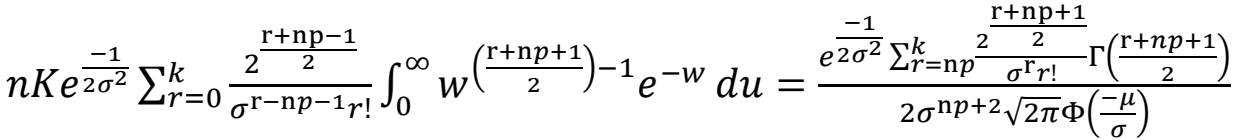

Thus,

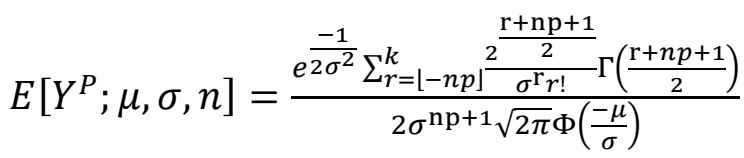

And

$$
\begin{aligned}
& \mu_{j}(\mu, \sigma, n)=E\left[(Y-\mu)^{j} ; \mu, \sigma, n\right] \\
& =\sum_{p=0}^{j-1}(-1)^{j-p}\left(\begin{array}{c}
j \\
j-p
\end{array}\right) \mu^{j-p} E\left[Y^{P} ; \mu, \sigma, n\right]+E\left[Y^{j} ; \mu, \sigma, n\right] \\
& =\sum_{p=0}^{j-1}(-1)^{j-p}\left(\begin{array}{c}
j \\
j-p
\end{array}\right) \mu^{j-p} \frac{e^{\frac{-1}{2 \sigma^{2}} \sum_{r=[-n p]}^{k} \frac{2^{\frac{\mathrm{r}+\mathrm{np}+1}{2}}}{\sigma^{\mathrm{r}} r !}} \Gamma\left(\frac{\mathrm{r}+n p+1}{2}\right)}{2 \sigma^{\mathrm{n} p+2} \sqrt{2 \pi} \Phi\left(\frac{-\mu}{\sigma}\right)}
\end{aligned}
$$

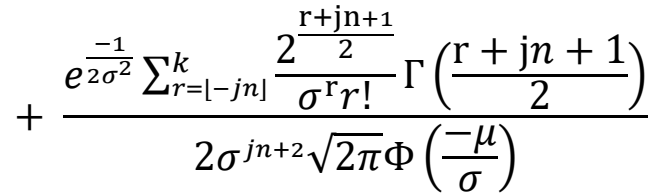

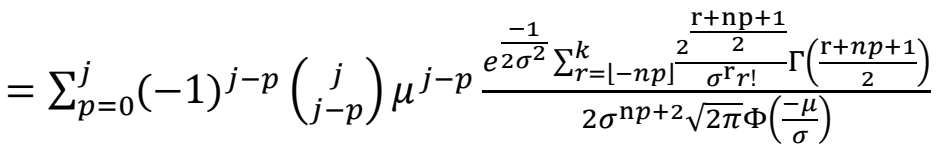

where $\lfloor x\rfloor$ is the greatest integer function less than $x$.

It is important to observe that in particular, in equation (9), if we taken $=-1$, then $g$ is an inverse transform of $f$ and by putting $k=7, \mu=1$ and evaluating $E\left[Y^{P} ; 1, \sigma,-1\right]$ at $p=1,2$ respectively, we obtain the result in [8]. 


\section{Remark 4.1 Furthermore observe that:}

(1). Iwueze (2007), for $\mu=1, n=1$, the authors expressed $E[Y]$ in terms of cumulative distribution function of the standard normal distribution and $E\left[(Y-1)^{2}\right]$ in terms of cumulative distribution function of the standard normal distribution and Chi-square distribution function.

(2). Nwosu, Iwueze and Ohakwe (2010), for $\mu=1, n=-1$, the authors expressed $E[Y]$ and $E\left[(Y-1)^{2}\right]$ in terms of cumulative distribution function of the standard normal distribution and Gamma distribution function.

(3). Ohakwe, Dike and Akpanta (2012), for $\mu=1, n=2$, the authors expressed $E[Y]$ and $E\left[(Y-1)^{2}\right]$ in terms of cumulative distribution function of the standard normal distribution.

(4). Nwosu, Iwueze, and Ohakwe. (2013), for $\mu=1, n=-1$, the authors expressed $E[Y]$ and $E\left[(Y-1)^{2}\right]$ in terms of cumulative distribution function of the standard normal distribution and Chi-square distribution function.

(5). Ibeh and Nwosu (2013), for $\mu=1, n=-2$, the authors expressed $E[Y]$ and $E\left[(Y-1)^{2}\right]$ in terms of cumulative distribution function of the standard normal distribution and Chi-square distribution function.

(6). Ajibade, Nwosu and Mbaegbu (2015), for $\mu=1, n=\frac{-1}{2}$, the authors expressed $E[Y]$ and $E\left[(Y-1)^{2}\right]$ in terms of cumulative distribution function of the standard normal distribution and Chi-square distribution function.

Hence, it suffices to say that the expression for the moments is by no means unique.

Furthermore, the aforementioned authors above seems to be somewhat restrictive in their estimation of moments; they all estimated only for the first moment about the origin (mean) and the second central moment (variance). Hence, in this paper such restriction is dispensed with. 
The Moment Generating Function Associated with $g(y ; \mu, \sigma, n)$ and $f_{T}(x ; \mu, \sigma)$

The moment generating function of $Y$ is given by

$$
M_{Y}(t ; \mu, \sigma, n)=E\left(e^{t Y} ; \mu, \sigma, n\right)=\int_{0}^{\infty} e^{t y} g(y ; \mu, \sigma, n) d y=\int_{0}^{\infty} \sum_{i \geq 0}^{\infty} \frac{(t y)^{i}}{i !} g(y ; \mu, \sigma, n) d y
$$

Observe that the series $\sum_{r=0}^{\infty} \frac{(t y)^{i}}{i !}$ converges uniformly (by ratio test) [3,13], hence by Taylors series expansion, for some positive constant $l$ (sufficiently large enough), there exists a number $\rho\left(i_{l}\right)$ between 0 and $t y$ such that $\rho\left(i_{l}\right) \longrightarrow 0$ as $i \rightarrow \infty[3,13]$, it then follows that as $i \rightarrow \infty$

$$
\int_{0}^{\infty} \sum_{i=0}^{k}\left(\frac{1}{i !}(t y)^{i}+\frac{1}{i !}(t y)\left(\rho\left(i_{l}\right)\right)^{i}\right) g(y ; \mu, \sigma, n) d y
$$

can be approximated by

$$
\begin{aligned}
& \int_{0}^{\infty} \sum_{i=0}^{l} \frac{1}{i !}(t y)^{i} g(y ; \mu, \sigma, n) d y=\sum_{i=0}^{l} \frac{t^{i}}{i !} \int_{0}^{\infty} y^{i} g(y ; \mu, \sigma, n) d y \\
& =\sum_{i=0}^{l} \frac{t^{i}}{i !} \int_{0}^{\infty} \frac{y^{i+\frac{1}{n}-1}}{|n| \sigma \sqrt{2 \pi} \Phi\left(\frac{-\mu}{\sigma}\right)} e^{\frac{-1}{2}\left(\frac{y^{\frac{1}{n}}-\mu}{\sigma}\right)^{2}} d y=\sum_{i=0}^{l} \frac{t^{i}}{i !} E\left[Y^{i} ; \mu, \sigma, n\right] \\
& =\sum_{i=0}^{l} \frac{t^{i}}{i !} \frac{e^{\frac{-1}{2 \sigma^{2}}} \sum_{r=\lfloor-n i]}^{k} \frac{2^{\frac{\mathrm{r}+\mathrm{n} i+1}{2}}}{\sigma^{\mathrm{r}} r !} \Gamma\left(\frac{\mathrm{r}+n i+1}{2}\right)}{2 \sigma^{\mathrm{n} i+2} \sqrt{2 \pi} \Phi\left(\frac{-\mu}{\sigma}\right)}
\end{aligned}
$$

For the moment generating function of $X$, recall that at $n=1, y=x$, it follows that $g(y ; \mu, \sigma, 1)=f_{T}(x ; \mu, \sigma)$. Hence

$$
\begin{aligned}
& M_{Y}(t ; \mu, \sigma, 1)=\int_{0}^{\infty} e^{t y} g(y ; \mu, \sigma, 1) d y=\int_{0}^{\infty} e^{t x} f_{T}(x ; \mu, \sigma) d x \\
& =E\left(e^{t X} ; \mu, \sigma\right)=M_{X}(t ; \mu, \sigma)=\sum_{i=0}^{l} \frac{t^{i}}{i !} \frac{e^{\frac{-1}{2 \sigma^{2}}} \sum_{r=\lfloor-i \mid}^{k} \frac{2^{\frac{\mathrm{r}+i+1}{2}}}{\sigma^{\mathrm{r}} r !} \Gamma\left(\frac{\mathrm{r}+i+1}{2}\right)}{2 \sigma^{i+2} \sqrt{2 \pi} \Phi\left(\frac{-\mu}{\sigma}\right)}
\end{aligned}
$$




\section{Existence of the Bell-Shape Curve Associated with $g(y ; \mu, \sigma, n)$ and $f_{T}(x ; \mu, \sigma)$}

Recall that $f_{T}(x ; \mu, \sigma)$, the left truncated normal distribution of $f$, which is given by

$$
f_{T}(x ; \mu, \sigma)=\frac{1}{\sigma \sqrt{2 \pi} \Phi\left(\frac{-\mu}{\sigma}\right)} e^{\frac{-1}{2}\left(\frac{x-\mu}{\sigma}\right)^{2}}, x \in R_{+}
$$

is normal distribution in the region $X>0$ with mean $\mu_{1}(\mu, \sigma, 1)$ and variance $\mu_{2}(\mu, \sigma, 1)$, where

$$
\begin{gathered}
\mu_{1}(\mu, \sigma, 1)=\frac{e^{\frac{-1}{2 \sigma^{2}} \sum_{r=[-j n]}^{k} \frac{2^{\frac{\mathrm{r}+2}{2}}}{\sigma^{\mathrm{r}} r !} \Gamma\left(\frac{\mathrm{r}+2}{2}\right)}}{2 \sigma^{3} \sqrt{2 \pi} \Phi\left(\frac{-\mu}{\sigma}\right)} \\
\mu_{2}(\mu, \sigma, 1)=\sum_{p=0}^{2}(-1)^{2-p}\left(\begin{array}{c}
2 \\
2-p
\end{array}\right) \mu^{2-p} \frac{e^{\frac{-1}{2 \sigma^{2}} \sum_{r=l-p]}^{k} \frac{2^{\frac{\mathrm{r}+p+1}{2}}}{\sigma^{\mathrm{r}} r !} \Gamma\left(\frac{\mathrm{r}+p+1}{2}\right)}}{2 \sigma^{p+2} \sqrt{2 \pi} \Phi\left(\frac{-\mu}{\sigma}\right)}
\end{gathered}
$$

If we denote this mean and variance of the truncated normal distribution $f_{T}(x ; \mu, \sigma)$ by $\mu_{T}$ and $\sigma_{T}^{2}$ (i.e. $\mu_{T}=\mu_{1}(\mu, \sigma, 1)$ and $\left.\sigma_{T}^{2}=\mu_{2}(\mu, \sigma, 1)\right)$. It is well known that the shape of $f_{T}(x ; \mu, \sigma)$ varies as the value of $\sigma_{T}^{2}$ varies (consequently as $\sigma$ varies since $\sigma_{T}^{2}$ depend on $\sigma$ ), hence $\sigma$ is also the shape parameter for $f_{T}(x ; \mu, \sigma)$.

Also recall that $g(y ; \mu, \sigma, n)$, the generalized power transformation of $f_{T}(x ; \mu, \sigma)$, which is given by

$$
g(y ; \mu, \sigma, n)=\left\{\begin{array}{c}
\frac{y^{\frac{1}{n}-1}}{|n| \sigma \sqrt{2 \pi} \Phi\left(\frac{-\mu}{\sigma}\right)} e^{\frac{-1}{2}\left(\frac{y^{\frac{1}{n}}-\mu}{\sigma}\right)^{2}}, y \in R_{+}, n \in \mathcal{F} . \\
0 \text { otherwise }
\end{array}\right.
$$

Is normal distribution in the region $X>0$ with mean $\mu_{1}(\mu, \sigma, n)$ and variance $\mu_{2}(\mu, \sigma, n)$, where

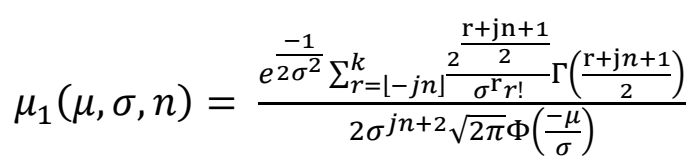

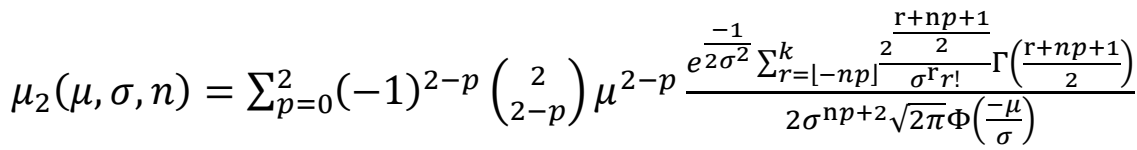

If we denote this mean and variance of the generalized $n$-power transform of $f_{T}(x ; \mu, \sigma)$ by $\mu_{T}(n)$ and $\sigma_{T}^{2}(n)$ (i.e. $\mu_{T}(n)=\mu_{1}(\mu, \sigma, n)$ and $\sigma_{T}^{2}(n)=\mu_{2}(\mu, \sigma, n)$ ). It follows that for every 
fixed $n \in R$, the shape of $g(y ; \mu, \sigma, n)$ varies as the value of $\sigma_{T}^{2}(n)$ varies (consequently as $\sigma$ varies since $\sigma_{T}^{2}(n)$ depend on $\left.\sigma\right)$, hence $\sigma$ is also the shape parameter for $g(y ; \mu, \sigma, n)$. Observe that. $\mu_{T}(1)=\mu_{1}(\mu, \sigma, 1)=\mu_{T}$ and $\sigma_{T}^{2}(1)=\mu_{2}(\mu, \sigma, 1)=\sigma_{T}^{2}$.

Now, we observe that $\sigma_{T}^{2}(n)$ (and $\sigma_{T}^{2}$ ) depend on $\sigma$. A common research interest of several authors (see $[2,6-11,14]$ ) is to find the value of $\sigma$ for which $\mu_{T}(1)=\mu_{T}(n)$ for every fixed $n \neq 1(n \in R)$. This is the so-called normality condition. Furthermore, it is expected that at this point $\sigma_{T}^{2}(1)=\sigma_{T}^{2}(n)$ for every fixed $n \neq 1(n \in R)$. Observe that $g(y ; \mu, \sigma, n)$ and $f_{T}(x ; \mu, \sigma)$ are strictly monotone and have one turning point, furthermore $g(y ; \mu, \sigma, n)>0$ and $f_{T}(x ; \mu, \sigma)>0$ for every $x, y \in R_{+}$, and for a fixed $n \in \mathcal{F}$. Which implies that the values of $x, y$ at these turning points maximizes $f_{T}(x ; \mu, \sigma), g(y ; \mu, \sigma, n)$ respectively. Consequently, by classical calculus, it is well known that these values of $x, y$ at this turning point coincide with the mode of $f_{T}(x ; \mu, \sigma), g(y ; \mu, \sigma, n)$ respectively. We shall determine this values of $x, y$ using the Rolle's theorem. Now we state the following theorem which is equivalent to the (socalled) normality condition.

\section{Theorem 6.1}

Let $f_{T}\left(x ; \mu_{T}, \sigma_{T}\right)$ be a truncated normal distribution and $g\left(y ; \mu_{T}(n), \sigma_{T}\left(n_{0}\right), n_{0}\right)$ the generalized $n_{0}$-power transformation of $f_{T}\left(x ; \mu_{T}, \sigma_{T}\right)$ induced by $y=x^{n_{0}}$, then $g\left(y ; \mu_{T}\left(n_{0}\right), \sigma_{T}\left(n_{0}\right), n_{0}\right)$ has a Bell-shape that coincide with $f_{T}\left(x ; \mu_{T}, \sigma_{T}\right)$ if there exists a sequence $\left\{\sigma_{j}\right\}_{j=1}^{\infty} \subset\left(\beta_{1}, \beta_{2}\right) \subset R_{+}$and at least one point $\sigma_{0} \in\left(\beta_{1}, \beta_{2}\right)$ such that the $\left\{\sigma_{j}\right\}_{j=1}^{\infty}$ converges to $\sigma_{0} \in\left(\beta_{1}, \beta_{2}\right)$ (i.e. $\sigma_{j} \rightarrow \sigma_{0}$ as $j \rightarrow \infty$ ) and $\sigma_{0}$ is a zero solution to the problem

$$
\text { maximize: } g\left(y ; \mu_{T}\left(n_{0}\right), \sigma_{T}\left(n_{0}\right), n_{0}\right)
$$

$$
\text { at the point: } y=x_{0}
$$

provided, $f_{T}\left(x ; \mu_{T}, \sigma_{T}\right) \leq f_{T}\left(x_{0} ; \mu_{T}, \sigma_{T}\right) \forall x \in R_{+}$.

\section{Proof.}

Observe that $f_{T}\left(x ; \mu_{T}, \sigma_{T}\right)$ is bounded above and continuous, hence by boundedness above, it follows there exist a positive constant $C$ such that

$$
f_{T}\left(x ; \mu_{T}, \sigma_{T}^{*}\right) \leq C \forall x \in R_{+}
$$

and by continuity in $R_{+}$, it follows that there exists a constant $u_{0} \in R_{+}$such that $C=$ $\operatorname{Supf}_{T}\left(u_{0} ; \mu_{T}, \sigma_{T}\right)$, hence we must have $u_{0}=x_{0}$. This justisfy the existence of such $x_{0}$. 
Hence the problem is equivalent to

$$
\begin{gathered}
\text { maximize: } g\left(y ; \mu_{T}\left(n_{0}\right), \sigma_{T}\left(n_{0}\right), n_{0}\right) \\
\text { at the point: } y=u_{0}
\end{gathered}
$$

Now, suppose for contradiction that there is no such $\sigma \in R_{+}$(recall that $\sigma_{T}$ is a function of $\sigma$, i.e. $\sigma_{T}$ depend on $\sigma$ ) that satisfies the maximization problem. This implies that for every $\sigma \in R_{+}$, the maximization problem becomes

$$
\begin{gathered}
\text { maximize: } g\left(y ; \mu_{T}\left(n_{0}\right), \sigma_{T}\left(n_{0}\right), n_{0}\right) \\
\text { at the point: } y \neq u_{0}
\end{gathered}
$$

If $y \neq u_{0}$, it implies that there is an $\varepsilon \neq 0$ such that $y=u_{0} \pm \varepsilon$, hence the maximization problem becomes

$$
\begin{aligned}
& \text { maximize: } g\left(y ; \mu_{T}\left(n_{0}\right), \sigma_{T}\left(n_{0}\right), n_{0}\right) \\
& \text { at the point: } y=u_{0} \pm \varepsilon
\end{aligned}
$$

It then follows that

$$
C=\operatorname{Sup}\left\{f_{T}\left(u_{0} \pm \varepsilon ; \mu_{T}, \sigma_{\dot{T}}\right): \forall \varepsilon \neq 0\right\} \Rightarrow \Longleftarrow .
$$

Observe that this is a contradiction to the maximality of $C$ at $u_{0}$ since $\varepsilon \neq 0$. And converly, if the maximality conditionof $C$ holds, it

$$
\begin{gathered}
\Rightarrow\left\{f_{T}\left(u_{0} \pm \varepsilon ; \mu_{T}, \sigma_{T}\right): \forall \varepsilon \neq 0\right\}<C \\
\Rightarrow f_{T}\left(u_{0} ; \mu_{T}, \sigma_{T}\right) \leq C \text { for } \varepsilon=0 \\
\Rightarrow \operatorname{Supf}_{T}\left(u_{0} ; \mu_{T}, \sigma_{T}\right)=C
\end{gathered}
$$

This contradict the fact that $\varepsilon \neq 0$.

Thus we must have that there is at least one $\sigma \in R_{+}$(for such $\sigma \in R_{+}, \varepsilon=0$ ) that satisfies the maximization problem. This completes the proof.

We now proceed to solve the maximization problem of equation (16) and equation (17) which is equivalent to the maximization problem of equation (14) and equation (15).

Clearly $g\left(y ; \mu_{T}\left(n_{0}\right), \sigma_{T}\left(n_{0}\right), n_{0}\right)$ is differentiable in the given subset $D$ of $R_{+}$and by classical optimization theory of calculus, a necessary condition for existence of maximum (extreme) point of $g\left(y ; \mu_{T}\left(n_{0}\right), \sigma_{T}\left(n_{0}\right), n_{0}\right)$ is that the derivatives of $g\left(y ; \mu_{T}\left(n_{0}\right), \sigma_{T}\left(n_{0}\right), n_{0}\right)$ must be equal to zero $[3,13,15]$. This implies that

$$
\frac{d g\left(y ; \mu_{T}\left(n_{0}\right), \sigma_{T}\left(n_{0}\right), n_{0}\right)}{d y}=0
$$

We now proceed to solve for equation (22). Observe that 
$\frac{d g\left(y ; \mu_{T}\left(n_{0}\right), \sigma_{T}\left(n_{0}\right), n_{0}\right)}{d y}=K\left[\left(\frac{1}{n_{0}}-1\right) y^{\frac{1}{n_{0}}-2} e^{\frac{-1}{2}\left(\frac{y^{\frac{1}{n_{0}}}-\mu}{\sigma}\right)^{2}}-\right.$
$\left.y^{\frac{2}{n_{0}}-2} \frac{1}{n_{0}}\left(\frac{y^{\frac{1}{n_{0}}}-\mu}{\sigma^{2}}\right) e^{\frac{-1}{2}\left(\frac{y^{\frac{1}{n_{0}}}}{\sigma}\right)^{2}}\right]=K y^{\frac{1}{n_{0}}-2} e^{\frac{-1}{2}\left(\frac{y^{\frac{1}{n_{0}}}-\mu}{\sigma}\right)^{2}}\left[\left(\frac{1}{n_{0}}-1\right)-y^{\frac{1}{n_{0}}} \frac{1}{n_{0}}\left(\frac{y^{\frac{1}{n_{0}}}-\mu}{\sigma^{2}}\right)\right]$

By equation (22) it follows that

$$
K y^{\frac{1}{n_{0}}-2} e^{\frac{-1}{2}\left(\frac{y^{\frac{1}{n_{0}}}-\mu}{\sigma}\right)^{2}}\left[\left(\frac{1}{n_{0}}-1\right)-y^{\frac{1}{n_{0}}} \frac{1}{n_{0}}\left(\frac{y^{\frac{1}{n_{0}}}-\mu}{\sigma^{2}}\right)\right]=0 .
$$

Since $K y^{\frac{1}{n_{0}}-2} e^{\frac{-1}{2}\left(\frac{y^{\frac{1}{n_{0}}}-\mu}{\sigma}\right)^{2}}>0 \forall y \in R_{+}$, we must have that

$$
\left(\frac{1}{n_{0}}-1\right)-y^{\frac{1}{n_{0}}} \frac{1}{n_{0}}\left(\frac{y^{\frac{1}{n_{0}}}-\mu}{\sigma^{2}}\right)=0
$$

By simplifying the above equation we have

$$
\sigma^{2}\left(1-n_{0}\right)-y^{\frac{2}{n_{0}}}+\mu y^{\frac{1}{n_{0}}}=0
$$

Now if we take $v=y^{\frac{1}{n_{0}}}$, we obtain

$$
v^{2}-\mu v-\sigma^{2}\left(1-n_{0}\right)=0
$$

and if we take $v=y^{\frac{-1}{n_{0}}}$, we obtain

$$
\sigma^{2}\left(1-n_{0}\right) v^{2}+\mu v-1=0
$$

Thus, the solution to equation (23) and equation (24) is given by

$$
v=\left\{\begin{array}{l}
\frac{\mu \pm \sqrt{\mu^{2}-4 \sigma^{2}\left(n_{0}-1\right)}}{2} \\
\frac{\mu \pm \sqrt{\mu^{2}-4 \sigma^{2}\left(n_{0}-1\right)}}{2 \sigma^{2}\left(n_{0}-1\right)}
\end{array}\right.
$$

where $\left(\frac{\mu}{2 \sigma}\right)^{2}>n_{0}-1$. 
Solutions relating to equation (24) have been given by virtually all the authors mentioned above for specific value of $n_{0}$ and $\mu$. Using equation (23), we have that $v=y_{\max }^{\frac{1}{n_{0}}}$. Now, by equation (17) it follows that $u_{0}=y_{\max }=\mu$. Thus,

$$
v^{2}-u_{0} v-\sigma^{2}\left(1-n_{0}\right)=0 \text { ifv }=u_{0}^{\frac{1}{n_{0}}}
$$

And

$$
\sigma^{2}\left(1-n_{0}\right) v^{2}+u_{0} v-1=0 \text { if } v=u_{0}^{\frac{-1}{n_{0}}}
$$

If we putz $z_{0}=u_{0}^{\frac{1}{n_{0}}}$ and $w_{0}=u_{0}^{\frac{-1}{n_{0}}}$, then we have

$$
G(\sigma)=0 ; G(\sigma)=z_{0}^{2}-u_{0} z_{0}+\sigma^{2}\left(n_{0}-1\right)
$$

And

$$
H(\sigma)=0 ; H(\sigma)=-\sigma^{2}\left(n_{0}-1\right) w_{0}^{2}+u_{0} w_{0}-1
$$

This reduces to solving for the zero of the functions $G(\sigma)$ and $H(\sigma)$.

For $G(\sigma)$, this implies that given $0 \leq \delta_{1}<\delta_{2}$, if we take $\sigma_{a}=\sqrt{\frac{u_{0} z_{0}-z_{0}{ }^{2}-\delta_{1}}{n_{0}-1}}$ and $\sigma_{b}=$ $\sqrt{\frac{u_{0} z_{0}-z_{0}^{2}+\delta_{2}}{n_{0}-1}}$, then $G\left(\sqrt{\frac{u_{0} z_{0}-z_{0}^{2}-\delta_{1}}{n_{0}-1}}\right)=-\delta_{1} \leq 0$ and $G\left(\sqrt{\frac{u_{0} z_{0}-z_{0}^{2}+\delta_{2}}{n_{0}-1}}\right)=\delta_{2}>0$

It follows that

$$
G\left(\sqrt{\frac{u_{0} z_{0}-z_{0}^{2}-\delta_{1}}{n_{0}-1}}\right) G\left(\sqrt{\frac{u_{0} z_{0}-z_{0}^{2}+\delta_{2}}{n_{0}-1}}\right)=-\delta_{1} \delta_{2}<0 \text { if } \delta_{1} \neq 0
$$

This implies that there exists a sequence $\left\{\sigma_{j}\right\}_{j=1}^{\infty} \subset\left(\sigma_{a}, \sigma_{b}\right)$ and at least one point $\sigma_{0} \in$ $\left(\sigma_{a}, \sigma_{b}\right)$ such that the $\left\{\sigma_{j}\right\}_{j=1}^{\infty}$ converges to $\sigma_{0} \in\left(\sigma_{a}, \sigma_{b}\right)$ (i.e. $\left.\sigma_{j} \rightarrow \sigma_{0} a s j \rightarrow \infty\right)$ and $G\left(\sigma_{0}\right)=$ 0

For $H(\sigma)$, this implies that given $\gamma_{1}=0$ and $\gamma_{2}>0$, if we take $\sigma_{p}=\sqrt{\frac{\left(u_{0} w_{0}+\gamma_{1}\right)}{\left(n_{0}-1\right) w_{0}^{2}}}$ and $\sigma_{q}=$ $\sqrt{\frac{\left(u_{0} w_{0}+\gamma_{2}\right)}{\left(n_{0}-1\right) w_{0}{ }^{2}}}$, then $H\left(\sqrt{\frac{\left(u_{0} w_{0}+\gamma_{1}\right)}{\left(n_{0}-1\right) w_{0}^{2}}}\right)=-1<0$ and $H\left(\sqrt{\frac{\left(u_{0} w_{0}-1-\gamma_{2}\right)}{\left(n_{0}-1\right) w_{0}^{2}}}\right)=\gamma_{2}>0$

It follows that

$$
H\left(\sqrt{\frac{u_{0} w_{0}+\gamma_{1}}{\left(n_{0}-1\right) w_{0}^{2}}}\right) H\left(\sqrt{\frac{u_{0} w_{0}-1-\gamma_{2}}{\left(n_{0}-1\right) w_{0}^{2}}}\right)=-\gamma_{2}<0
$$


This implies that there exists a sequence $\left\{\sigma_{i}\right\}_{i=1}^{\infty} \subset\left(\sigma_{p}, \sigma_{q}\right)$ and at least one point $\sigma_{0} \in\left(\sigma_{p}, \sigma_{q}\right)$ such that the sequence $\left\{\sigma_{i}\right\}_{i=1}^{\infty}$ converges to $\sigma_{0} \in\left(\sigma_{p}, \sigma_{q}\right)$ (i.e. $\sigma_{i} \rightarrow \sigma_{0}$ asi $\rightarrow \infty$ ) and $H\left(\sigma_{0}\right)=0$ [1]. This completes the proof.

$\left(\sigma_{a}, \sigma_{b}\right)$ and $\left(\sigma_{p}, \sigma_{q}\right)$ are intervals of normality corresponding to equation (23) and equation (24). This is the so-called interval of normality estimated by above mentioned authors using the Monte carol simulation method.

Furthermore, it follows from equation (25), that we can define the functions $G$ and $H$ as such

$$
G(\sigma)=\mu-2 z_{0}+\sqrt{\mu^{2}-4 \sigma^{2}\left(n_{0}-1\right)}
$$

$$
H(\sigma)=\mu-2 \sigma^{2}\left(n_{0}-1\right) w_{0}+\sqrt{\mu^{2}-4 \sigma^{2}\left(n_{0}-1\right)}
$$

Also, equation (26) and equation (27) are nonlinear problems of finding the zero(s) of $G$ and $H$ for every given value of $\mu$, which can be solved using any of the iteration formula for finding the zero(s) (i.e. root) of a nonlinear equations [4].

\section{CONCLUSION}

Having considered various transformation problems for a left-truncated normal distribution as announced by these researchers in the literature, particularly for a random variable that follows a left-truncated normal distribution, we obtained the probability distribution function of $g(y ; \mu, \sigma, n)$, called the generalized power transformation of $f_{T}(x ; \mu, \sigma)$, which is a generic form of all the distributions studied by above mentioned researchers. Also, moments associated with the distribution are similarly obtained, as denoted by $\mu_{1}(\mu, \sigma, n)$ and $\mu_{2}(\mu, \sigma, n)$. In particular, in equation (25), if we take $\mu=1, n=n_{0}=-2, \frac{-1}{2}$; as assumed by the authors in $[6,9]$ for a multiplicative time series model, we obtain the corresponding expressions for their $y_{\max }$ respectively. Furthermore, we employed the concept of approximation theory to establish the existence of $y_{\max }$ in the interval $\left(\sigma_{a}, \sigma_{b}\right)\left(\left(\sigma_{p}, \sigma_{q}\right)\right)$ corresponding to equation (23) and equation (24). This is the so-called interval of normality estimated by above mentioned authors using the Monte carol simulation method. Thus, the results presented in this research, actually unify and as well trivialized the results recently since particularly as we varies the value of $n$ in $g(y ; \mu, \sigma, n)$ we obtain the corresponding results of the above named researcher.

\section{REFERENCES}

[1] Ahmed S. E. et al. (2010). A Truncated Version of the Birnbaum-Sauders Distribution with Application in Financial Risk. Pakistani Journal of Statistics: 26(1), 293-311.

[2] Ajibade, Bright F., Nwosu, Chinwe R., and Mbaegbu, J. I. (2015). The Distribution of the Inverse Square Root Transformed Error Component of the Multiplicative Time Series Model. Journal of Modern Applied Statistical Methods: 14 (2), Article 15. 
[3] Bagui, S. C. and Mehra, K. L. (2016). Convergence of Binomial, Poisson, NegativeBinomial and Gamma to Normal Distribution: Moment Generating Functions Technique. American Journal of Mathematics and Statistics: .6(3), 115-121. DOI:10.5923/j.ajms.20160603.05.

[4] Ferng. R. (1997). Lecture Notes on Numerical Analysis: National Chiao Tung University, Hsin-Chu, Taiwan.

[5] George G. Roussas, (2007). A Course in Mathematical Statistics: Second Edition, Academic Press, USA.

[6] Ibeh, G. C and Nwosu, C. R. (2013).Study on the Error Component of Multiplicative Time Series Model under Inverse Square Transformation. American Journal of Mathematics and Statistics: 3(6): 362-374 DOI: 10.5923/j.ajms.20130306.10.

[7] Iwueze Iheanyi S. (2007). Some Implications of Truncating the $N(1, \sigma 2)$ Distribution to the left at Zero. Journal of Applied Sciences: 7(2), 189-195.

[8] Nwosu C. R, Iwueze I.S. and Ohakwe J. (2010). Distribution of the Error Term of the Multiplicative Time Series Model Under Inverse Transformation. Advances and Applications in Mathematical Sciences. 7(2), 119 - 139.

[9] Nwosu, C.R., Iwueze, I.S., \& Ohakwe J. (2013). Condition for Successful Inverse Transformation of the Error Component of the Multiplicative Time Series Model. Asian Journal of Applied Science: 6(1), 1-15. doi:10.3923/ajaps.2013.1.15.

[10] Ohakwe J., Dike O. A and Akpanta A.C. (2012). The Implication of Square Root Transformation on a Gamma Distributed Error Component of a Multiplicative Time Series model. Proceedings of African Regional Conference on Sustainable Development on (pp. $65-78)$. University of Calabar, Nigeria.

[11] Ohakwe, J., Iwuoha, O., and Otuonye, E. L. (2013). Condition for Successful Square Transformation in Time Series Modeling. Applied Mathematics: Vol. (4), 680-687.

[12] Okasha, M.K. and Iyad M. A. (2014). Inference on the Doubly Truncated Gamma Distribution for Lifetime Data. International Journal of Mathematics and Statistics Invention: 2(11), (IJMSI) E-ISSN: 2321 - 4767.

[13] Osisiogu, U. A. (1998). An Introduction to Real Analysis: Bestsort Educational Book. Nigeria.

[14] Otuonye, E. L., Iwueze, I. S., \& Ohakwe, J. (2011). The Effect of Square Root Transformation on the Error Component of the Multiplicative Time Series Model. International Journal of Statistics and Systems: 6(4), 461-476.

[15] Spiegel, M.R, Schiller J.J. and Srinivasan, R. A. (2000). Probability and Statistics: Second Edition, McGraw Hill Companies Inc., USA. 